\title{
Some New Classes of Generalized Difference Strongly Summable $n$-Normed Sequence Spaces Defined by Ideal Convergence and Orlicz Function
}

\author{
Adem Kılıçman ${ }^{1}$ and Stuti Borgohain ${ }^{2}$ \\ ${ }^{1}$ Department of Mathematics and Institute for Mathematical Research, University of Putra Malaysia, \\ 43400 Serdang, Selangor, Malaysia \\ ${ }^{2}$ Department of Mathematics, Indian Institute of Technology Bombay, Powai, Mumbai, Maharashtra 400076, India
}

Correspondence should be addressed to Adem Kılıçman; akilic@upm.edu.my

Received 23 January 2014; Accepted 1 July 2014; Published 23 July 2014

Academic Editor: Feyzi Başar

Copyright (C) 2014 A. Kılıçman and S. Borgohain. This is an open access article distributed under the Creative Commons Attribution License, which permits unrestricted use, distribution, and reproduction in any medium, provided the original work is properly cited.

\begin{abstract}
We study some new generalized difference strongly summable $n$-normed sequence spaces using ideal convergence and an Orlicz function in connection with de la Vallèe Poussin mean. We give some relations related to these sequence spaces also.
\end{abstract}

\section{Introduction}

Let $\ell_{\infty}, c$, and $c_{0}$ be the Banach space of bounded, convergent, and null sequences $x=\left(x_{k}\right)$, respectively, with the usual norm $\|x\|=\sup _{n}\left|x_{n}\right|$.

A sequence $x \in \ell_{\infty}$ is said to be almost convergent if all of its Banach limits coincide.

Let $\widehat{c}$ denote the space of all almost convergent sequences.

Lorentz in [1] proved that

$$
\widehat{c}=\left\{x \in l_{\infty}: \lim _{m} t_{m, n}(x) \text { exists uniformly in } n\right\},
$$

where

$$
t_{m, n}(x)=\frac{x_{n}+x_{n+1}+\cdots+x_{m+n}}{m+1} .
$$

The following space of strongly almost convergent sequence was introduced by Maddox in [2]:

$$
\begin{aligned}
& {[\widehat{c}]=\left\{x \in \ell_{\infty}: \lim _{m} t_{m, n}(|x-L e|)\right.} \\
&\text { exists uniformly in } n \text { for some } L\},
\end{aligned}
$$

Let $\sigma$ be a one-to-one mapping from the set of positive integers into itself such that $\sigma^{m}(n)=\sigma^{m-1}(\sigma(n)), m=$ $1,2,3, \ldots$, where $\sigma^{m}(n)$ denotes the $m$ th iterative of the mapping $\sigma$ in $n$.

A continuous linear functional $\phi$ on $\ell_{\infty}$ is said to be an invariant mean or a $\sigma$-mean, if and only if it satisfies the following conditions:

(1) $\phi(x) \geq 0$, when the sequence $x=\left(x_{n}\right)$ is such that $x_{n} \geq 0$ for all $n$;

(2) $\phi(e)=1$, where $e=(1,1, \ldots)$;

(3) $\phi\left(x_{\sigma(n)}\right)=\phi(x)$, for all $x \in \ell_{\infty}$.

For a certain kind of mapping $\sigma$, we get that every invariant mean $\phi$ extends the functional limit on the space $c$, such that $\phi(x)=\lim x$ for all $x \in c$. Consequently, we get that $c \subset V_{\sigma}$, where $V_{\sigma}$ is the set of bounded sequences with equal $\sigma$-means.

Schaefer in [3] proved that

$$
\begin{gathered}
V_{\sigma}=\left\{x \in \ell_{\infty}: \lim _{k} t_{k m}(x)=L \text { uniformly in } m\right. \text { for some } \\
L=\sigma-\lim x\},
\end{gathered}
$$

where $e=(1,1, \ldots)$. 
where

$$
t_{k m}(x)=\frac{x_{+} x_{\sigma(m)}+\cdots+x_{\sigma^{k}(m)}}{k+1}, \quad t_{-1, m}=0
$$

Thus we say that a bounded sequence $x=\left(x_{k}\right)$ is $\sigma$ convergent, if and only if $x \in V_{\sigma}$ such that $\sigma^{k}(n) \neq n$ for all $n \geq 0, k \geq 1$.

Note that similarly as the concept of almost convergence leads naturally to the concept of strong almost convergence, the $\sigma$-convergence leads naturally to the concept of strong $\sigma$ convergence.

A sequence $x=\left(x_{k}\right)$ is said to be strongly $\sigma$-convergent (Mursaleen [4]), if there exists a number $\ell$ such that

$$
\frac{1}{k} \sum_{i=1}^{k}\left|x_{\sigma^{i}(m)}-\ell\right| \longrightarrow 0, \quad \text { as } k \longrightarrow \infty \text { uniformly in } m \text {. }
$$

We write $\left[V_{\sigma}\right]$ to denote the set of all strong $\sigma$-convergent sequences and when (6) holds, we write $\left[V_{\sigma}\right]-\lim x=\ell$.

Taking $\sigma(m)=m+1$, we obtain $\left[V_{\sigma}\right]=[\widehat{c}]$. Then the strong $\sigma$-convergence generalizes the concept of strong almost convergence.

We also note that

$$
\left[V_{\sigma}\right] \subset V_{\sigma} \subset \ell_{\infty}
$$

The notion of ideal convergence was first introduced by Kostyrko et al. [5] as a generalization of statistical convergence which was later studied by many other authors.

An Orlicz function is a function $M:[0, \infty) \rightarrow[0, \infty)$, which is continuous, nondecreasing, and convex with $M(0)=$ $0, M(x)>0$, for $x>0$, and $M(x) \rightarrow \infty$, as $x \rightarrow \infty$.

Lindenstrauss and Tzafriri [6] used the idea of Orlicz function to construct the sequence space:

$$
\ell_{M}=\left\{\left(x_{k}\right) \in w: \sum_{k=1}^{\infty} M\left(\frac{|x|}{\rho}\right)<\infty \text {, for some } \rho>0\right\} .
$$

The space $\ell_{M}$ with the norm

$$
\|x\|=\inf \left\{\rho>0: \sum_{k=1}^{\infty} M\left(\frac{\left|x_{k}\right|}{\rho}\right) \leq 1\right\}
$$

becomes a Banach space which is called an Orlicz sequence space.

K1zmaz [7] studied the difference sequence spaces $\ell_{\infty}(\Delta)$, $c(\Delta)$, and $c_{0}(\Delta)$ of crisp sets. The notion is defined as follows:

$$
Z(\Delta)=\left\{x=\left(x_{k}\right):\left(\Delta x_{k}\right) \in Z\right\},
$$

for $Z=\ell_{\infty}, c$ and $c_{0}$, where $\Delta x=\left(\Delta x_{k}\right)=\left(x_{k}-x_{k+1}\right)$, for all $k \in N$.

The above spaces are Banach spaces, normed by

$$
\|x\|_{\Delta}=\left|x_{1}\right|+\sup _{k}\left|\Delta x_{k}\right|
$$

Later the idea of Kizmaz [7] was applied to introduce different types of difference sequence spaces and study their different properties by many others later on.
The generalized difference notion is defined as follows. For $m \geq 1$ and $n \geq 1$,

$Z\left(\Delta_{m}^{n}\right)=\left\{x=\left(x_{k}\right):\left(\Delta_{m}^{n} x_{k}\right) \in Z\right\}, \quad$ for $Z=\ell_{\infty}, c$ and $c_{0}$.

This generalized difference has the following binomial representation:

$$
\Delta_{m}^{n} x_{k}=\sum_{r=0}^{n}(-1)^{r}\left(\begin{array}{l}
n \\
r
\end{array}\right) x_{k+r m}
$$

The concept of 2-normed space was initially introduced by Gähler [8], in the mid of 1960s, while that of $n$-normed spaces can be found in Misiak [9]. Since then, many other authors have used this concept and obtained various results. Recently, several various activities have been initiated to study summability, sequence spaces, and related topics in these spaces. The notion of ideal convergence in 2-normed spaces was initially introduced by Gurdal [10]. Later on, it was extended to $n$-normed spaces by Gurdal and Sahiner in [11].

\section{Definitions and Preliminaries}

Let $n \in N$ and $X$ be a real vector space. A real valued function on $X^{n}$ satisfies the following four properties:

(1) $\left\|\left(z_{1}, z_{2}, \ldots, z_{n}\right)\right\|_{n}=0$ if and only if $z_{1}, z_{2}, \ldots, z_{n}$ are linearly dependent;

(2) $\left\|\left(z_{1}, z_{2}, \ldots, z_{n}\right)\right\|_{n}$ is invariant under permutation;

(3) $\left\|\left(z_{1}, z_{2}, \ldots, z_{n-1}, \alpha z_{n}\right)\right\|_{n}=|\alpha|\left\|z_{1}, z_{2}, \ldots, z_{n}\right\|_{n}$, for all $\alpha \in R$

(4) $\left\|\left(z_{1}, z_{2}, \ldots, z_{n-1}, x+y\right)\right\|_{n} \leq\left\|\left(z_{1}, z_{2}, \ldots, z_{n-1}, x\right)\right\|_{n}+$ $\left\|\left(z_{1}, z_{2}, \ldots, z_{n-1}, y\right)\right\|_{n}$

is called an $n$-norm on $X$ and the pair $\left(X,\|\cdot, \cdot\|_{n}\right)$ is called an $n$-normed space.

Let $X$ be a nonempty set. Then a family of sets $I \subseteq 2^{X}$ (power sets of $X$ ) is said to be an ideal if $I$ is additive that is $A, B \in I \Rightarrow A \cup B \in I$ and hereditary that is $A \in I, B \subseteq A \Rightarrow$ $B \in I$.

A sequence $\left(x_{k}\right)$ in a normed space $\left(X,\|\cdot, \cdot, \cdots\|_{n}\right)$ is said to be $I$-convergent to $x_{0} \in X$ with respect to $n$-norm, if for each $\varepsilon>0$, the set

$$
\begin{gathered}
E(\varepsilon)=\left\{k \in N:\left\|x_{k}-x_{0}, z_{1}, z_{2}, \ldots, z_{n-1}\right\|_{n} \geq \varepsilon,\right. \text { for every } \\
\left.z_{1}, z_{2}, \ldots, z_{n-1} \in X\right\} \text { belongs to } I .
\end{gathered}
$$

The generalized de la Vallée Poussin mean is defined by

$$
t_{n}(x)=\frac{1}{\lambda_{n}} \sum_{k \in I_{n}} x_{k}
$$

where $I_{n}=\left[n-\lambda_{n}+1, n\right]$ for $n=1,2 \ldots$ 
Then a sequence $x=\left(x_{k}\right)$ is said to be $(V, \lambda)$-summable to a number $L$, if $t_{n}(x) \rightarrow L$ as $n \rightarrow \infty$, and we write

$$
\begin{gathered}
{[V, \lambda]_{0}=\left\{x: \lim _{n} \frac{1}{\lambda_{n}} \sum_{k \in I_{n}}\left|x_{k}\right|=0\right\},} \\
{[V, \lambda]=\left\{x: x-\ell e \in[V, \lambda]_{0} \text { for some } \ell \in C\right\},} \\
{[V, \lambda]_{\infty}=\left\{x: \sup _{n} \frac{1}{\lambda_{n}} \sum_{k \in I_{n}}\left|x_{k}\right|<\infty\right\}}
\end{gathered}
$$

for the sets of sequences that are, respectively, strongly summable to zero, strongly summable, and strongly bounded by de la Vallée Poussin method.

Maddox introduced and studied the special case, where $\lambda_{n}=n$, for $n=1,2,3, \ldots$; the sets $[V, \lambda]_{0},[V, \lambda]$, and $[V, \lambda]_{\infty}$ reduce to the sets $w_{0}, w$, and $w_{\infty}$.

In this paper, we define some new sequence spaces in $n$-normed spaces by using Orlicz function with notion of generalized de la Vallèe Poussin mean, generalized difference sequences, and ideals. We will also introduce and examine certain new sequence spaces using the above tools as also the $n$-norm.

\section{Main Results}

Let $I$ be an admissible ideal of $N$, let $M$ be an Orlicz function, and let $\left(X,\|\cdot, \cdots\|_{n}\right)$ be a $n$-normed space. Further, let $s=$ $\left(s_{k}\right)$ be a bounded sequence of positive real numbers. By $S(n-X)$, we denote the space of all sequences defined over $\left(X,\|\cdot, \cdots\|_{n}\right)$.

In this paper, we have introduced the following sequence spaces:

$$
\begin{aligned}
& \left(\left[V_{\sigma}, \lambda, \Delta_{p}^{q}, M, r\right]_{0}^{I},\|\cdot, \cdot, \cdots\|_{n}\right) \\
& =\{x: \forall \varepsilon>0 \\
& \times\{n \in N: \\
& \frac{1}{\lambda_{n}} \sum_{k \in I_{n}}\left\{M\left(\frac{\left\|\Delta_{p}^{q} x_{\sigma^{k}(m)}, z_{1}, z_{2}, \ldots, z_{n-1}\right\|_{n}}{\rho}\right)\right\}^{r_{k}} \\
& \geq \varepsilon\} \in I\} \text {, }
\end{aligned}
$$

for some $\rho>0$ and each $z \in X$,

$$
\begin{aligned}
& \left(\left[V_{\sigma}, \lambda, \Delta_{p}^{q}, M, r\right]^{I},\|\cdot, \cdot, \cdots\|_{n}\right) \\
& =\{x: \forall \varepsilon>0 \\
& \quad \times\{n \in N: \\
& \quad \frac{1}{\lambda_{n}} \sum_{k \in I_{n}}\left\{M\left(\frac{\left\|\Delta_{p}^{q} x_{\sigma^{k}(m)}-L, z_{1}, z_{2}, \ldots, z_{n-1}\right\|_{n}}{\rho}\right)\right\}^{r_{k}} \\
& \geq \varepsilon\} \in I\},
\end{aligned}
$$

for some $\rho>0, L \in X$ and each $z \in X$,

$$
\begin{aligned}
& \left(\left[V_{\sigma}, \lambda, \Delta_{p}^{q}, M, r\right]_{\infty}^{I},\|\cdot, \cdot, \cdots\|_{n}\right) \\
& =\{x: \exists K>0, \\
& \text { s.t. }\left\{\sup _{n, m} \frac{1}{\lambda_{n}} \sum_{k \in I_{n}}\left\{M\left(\frac{\left\|\Delta_{p}^{q} x_{\sigma^{k}(m)}, z_{1}, z_{2}, \ldots, z_{n-1}\right\|_{n}}{\rho}\right)\right\}^{r_{k}}\right. \\
& \geq K\} \in I\},
\end{aligned}
$$

for some $\rho>0$ and each $z \in X$.

In particular, if we take $r_{k}=1$, for all $k$, we have

$$
\begin{aligned}
& \left(\left[V_{\sigma}, \lambda, \Delta_{p}^{q}, M\right]_{0}^{I},\|\cdot, \cdots\|_{n}\right) \\
& =\{x: \forall \varepsilon>0 \\
& \quad \times\{n \in N: \\
& \quad \frac{1}{\lambda_{n}} \sum_{k \in I_{n}}\left\{M\left(\frac{\left\|\Delta_{p}^{q} x_{\sigma^{k}(m)}, z_{1}, z_{2}, \ldots, z_{n-1}\right\|_{n}}{\rho}\right)\right\} \\
& \geq \varepsilon, \text { uniformly in } m\} \in I\},
\end{aligned}
$$

for some $\rho, \forall z_{1}, z_{2}, \ldots, z_{n-1} \in X$, 


$$
\begin{aligned}
& \left(\left[V_{\sigma}, \lambda, \Delta_{p}^{q}, M\right]^{I},\|\cdot, \cdot, \cdots\|_{n}\right) \\
& =\{x: \forall \varepsilon>0 \\
& \quad \times\{n \in N: \\
& \quad \frac{1}{\lambda_{n}} \sum_{k \in I_{n}}\left\{M\left(\frac{\left\|\Delta_{p}^{q} x_{\sigma^{k}(m)}-L, z_{1}, z_{2}, \ldots, z_{n-1}\right\|_{n}}{\rho}\right)\right\} \\
& \geq \varepsilon\} \in I\},
\end{aligned}
$$

for some $\rho>0, L \in X$ and each $z \in X$,

$$
\begin{aligned}
& \left(\left[V_{\sigma}, \lambda, \Delta_{p}^{q}, M\right]_{\infty}^{I},\|\cdot, \cdot, \cdots\|_{n}\right) \\
& =\{x: \exists K>0 \\
& \quad \times\left\{\sup _{n, m} \frac{1}{\lambda_{n}} \sum_{k \in I_{n}}\left\{M\left(\frac{\left\|\Delta_{p}^{q} x_{\sigma^{k}(m)}, z_{1}, z_{2}, \ldots, z_{n-1}\right\|_{n}}{\rho}\right)\right\}\right. \\
& \geq K\} \in I\} .
\end{aligned}
$$

Similarly, when $\sigma(m)=m+1$, then $\left(\left[\widehat{V}, \lambda, \Delta_{p}^{q}, M, r\right]_{0}^{I},\|\cdots\|_{n}\right)$, $\left(\left[V_{\sigma}, \lambda, \Delta_{p}^{q}, M, r\right]^{I},\|\cdots\|_{n}\right)$, and $\left(\left[V_{\sigma}, \lambda, \Delta_{p}^{q}, M, r\right]_{\infty}^{I},\|\cdots\|_{n}\right)$ are reduced to

$$
\begin{aligned}
& \left(\left[\widehat{V}, \lambda, \Delta_{p}^{q}, M, r\right]_{0}^{I},\|\cdots\|_{n}\right) \\
& =\{x: \forall \varepsilon>0 \\
& \quad \times\{n \in N: \\
& \quad \frac{1}{\lambda_{n}} \sum_{k \in I_{n}}\left\{M\left(\frac{\left\|\Delta_{p}^{q} x_{k+m}, z_{1}, z_{2}, \ldots, z_{n-1}\right\|_{n}}{\rho}\right)\right\}^{r_{k}} \\
& \geq \varepsilon\} \in I\},
\end{aligned}
$$

for some $\rho>0$ and each $z \in X$,

$$
\begin{aligned}
& \left(\left[\widehat{V}, \lambda, \Delta_{p}^{q}, M, r\right]^{I},\|\cdot, \cdots\|_{n}\right) \\
& =\{x: \forall \varepsilon>0 \\
& \times\{n \in N: \\
& \frac{1}{\lambda_{n}} \sum_{k \in I_{n}}\left\{M\left(\frac{\left\|\Delta_{p}^{q} x_{k+m}-L, z_{1}, z_{2}, \ldots, z_{n-1}\right\|_{n}}{\rho}\right)\right\}^{r_{k}} \\
& \geq \varepsilon\} \in I\}
\end{aligned}
$$

for some $\rho>0, L \in X$ and each $z \in X$,

$$
\begin{aligned}
& \left(\left[\widehat{V}, \lambda, \Delta_{p}^{q}, M, r\right]_{\infty}^{I},\|\cdot, \cdots\|_{n}\right) \\
& =\{x: \exists K>0, \\
& \text { s.t. }\left\{\sup _{n, m} \frac{1}{\lambda_{n}} \sum_{k \in I_{n}}\left\{M\left(\frac{\left\|\Delta_{p}^{q} x_{k+m}, z_{1}, z_{2}, \ldots, z_{n-1}\right\|_{n}}{\rho}\right)\right\}^{r_{k}}\right. \\
& \geq k\} \in I\},
\end{aligned}
$$

for some $\rho>0$, and each $z \in X$.

In particular, if we put $r_{k}=r$, for all $k$, then we have the spaces

$$
\begin{aligned}
\left(\left[\widehat{V}, \lambda, \Delta_{p}^{q}, M, r\right]_{0}^{I},\|\cdot, \cdots\|_{n}\right) & =\left(\left[\widehat{V}, \lambda, \Delta_{p}^{q}, M\right]_{0}^{I},\|\cdot, \cdot\|_{n}\right), \\
\left(\left[\widehat{V}, \lambda, \Delta_{p}^{q}, M, r\right]^{I},\|\cdot, \cdots\|_{n}\right) & =\left(\left[\widehat{V}, \lambda, \Delta_{p}^{q}, M\right]^{I},\|\cdot \cdot \cdot\|_{n}\right), \\
\left(\left[\widehat{V}, \lambda, \Delta_{p}^{q}, M, r\right]_{\infty}^{I},\|\cdot, \cdots\|_{n}\right) & =\left(\left[\widehat{V}, \lambda, \Delta_{p}^{q}, M\right]_{\infty}^{I},\|\cdot, \cdot\|_{n}\right) .
\end{aligned}
$$

Further when $\lambda_{n}=n$, for $n=1,2, \ldots$, the sets $\left(\left[\widehat{V}, \lambda, \Delta_{p}^{q}\right.\right.$, $\left.M]_{0}^{I},\|\cdot, \cdot,\|_{n}\right)$ and $\left(\left[\widehat{V}, \lambda, \Delta_{p}^{q}, M\right]^{I},\|\cdot, \cdot,\|_{n}\right)$ are reduced to $\left(\left[\widehat{c}_{0}\right.\right.$ $\left.\left.\left(M, \Delta_{p}^{q}\right)\right]^{I},\|\cdot, \cdot, \cdots\|_{n}\right)$ and $\left(\left[\widehat{c}\left(M, \Delta_{p}^{q}\right)\right]^{I},\|\cdot, \cdot, \cdots\|_{n}\right)$, respectively. 
Now, if we consider $M(x)=x$, then we can easily obtain

$$
\begin{aligned}
& \left(\left[V_{\sigma}, \lambda, \Delta_{p}^{q}, r\right]_{0}^{I},\|\cdot, \cdots\|_{n}\right) \\
& =\{x: \forall \varepsilon>0 \\
& \times\{n \in N: \\
& \frac{1}{\lambda_{n}} \sum_{k \in I_{n}}\left(\left\|\Delta_{p}^{q} x_{\sigma^{k}(m)}, z_{1}, z_{2}, \ldots, z_{n-1}\right\|_{n}\right)^{r_{k}} \\
& \geq \varepsilon \text {, uniformly in } m\} \in I\} \text {, } \\
& \left(\left[V_{\sigma}, \lambda, \Delta_{p}^{q}, r\right]^{I},\|\cdot, \cdots\|_{n}\right) \\
& =\{x: \forall \varepsilon>0 \\
& \times\{n \in N: \\
& \frac{1}{\lambda_{n}} \sum_{k \in I_{n}}\left(\left\|\Delta_{p}^{q} x_{\sigma^{k}(m)}-L, z_{1}, z_{2}, \ldots, z_{n-1}\right\|_{n}\right)^{r_{k}} \\
& \geq \varepsilon\} \in I\},
\end{aligned}
$$

for $L \in X$ and each $z \in X$,

$$
\begin{aligned}
& \left(\left[V_{\sigma}, \lambda, \Delta_{p}^{q}, r\right]_{\infty}^{I},\|\cdot, \cdots\|_{n}\right) \\
& =\{x: \exists K>0, \\
& \text { s.t. }\left\{\sup _{n, m} \frac{1}{\lambda_{n}} \sum_{k \in I_{n}}\left(\left\|\Delta_{p}^{q} x_{\sigma^{k}(m)}, z_{1}, z_{2}, \ldots, z_{n-1}\right\|_{n}\right)^{r_{k}}\right. \\
& \geq K\} \in I\} .
\end{aligned}
$$

If $x \in\left(\left[V_{\sigma}, \lambda, \Delta_{p}^{q}, M, r\right]^{I},\|\cdots\|_{n}\right)$, with $\left\{\left(1 / \lambda_{n}\right) \sum_{k \in I_{n}}\left\{M\left(\| \Delta_{p}^{q}\right.\right.\right.$ $\left.\left.\left.x_{\sigma^{k}(m)}-L, z_{1}, z_{2}, \ldots, z_{n-1} \|_{n} / \rho\right)\right\}^{r_{k}} \geq \varepsilon\right\} \in I$ as $n \rightarrow \infty$ uniformly in $m$, then we write $x_{k} \rightarrow L \in\left(\left[V_{\sigma}, \lambda, \Delta_{p}^{q}, M\right.\right.$, $\left.r]^{I},\|\cdots\|_{n}\right)$.

The following well-known inequality will be used later.
If $0 \leq r_{k} \leq \sup r_{k}=H$ and $C=\max \left(1,2^{H-1}\right)$, then

$$
\left|a_{k}+b_{k}\right|^{r_{k}} \leq C\left\{\left|a_{k}\right|^{r_{k}}+\left|b_{k}\right|^{r_{k}}\right\}
$$

for all $k$ and $a_{k}, b_{k} \in C$.

Lemma 1. Let $r_{k}>0$ and $s_{k}>0$. Then $c_{0}(s) \subset c_{0}(r)$, if and only if $\lim _{k \rightarrow \infty} \inf \left(r_{k} / s_{k}\right)>0$, where $c_{0}(r)=\left\{x:\left|x_{k}\right|^{r_{k}} \rightarrow\right.$ 0 as $k \rightarrow \infty$ \}.

Note that no other relation between $\left(r_{k}\right)$ and $\left(s_{k}\right)$ is needed in Lemma 1.

Theorem 2. Let $\lim _{k \rightarrow \infty}$ inf $r_{k}>0$. Then, $x_{k} \rightarrow$ L implies $x_{k} \rightarrow L \in\left(\left[V_{\sigma}, \lambda, \Delta_{p}^{q}, M, r\right]^{I},\|\cdots\|_{n}\right)$. Let $\lim _{k \rightarrow \infty} r_{k}=r>$ 0. If $x_{k} \rightarrow L \in\left(\left[V_{\sigma}, \lambda, \Delta_{p}^{q}, M, r\right]^{I},\|\cdots\|_{n}\right)$, then $L$ is unique.

Proof. Let $x_{k} \rightarrow$ L.

By the definition of Orlicz function, we have, for all $\varepsilon>0$,

$$
\begin{aligned}
& \left\{\lim _{n} \frac{1}{\lambda_{n}} \sum_{k \in I_{n}} M\left(\frac{\left\|\Delta_{p}^{q} x_{\sigma^{k}(m)}-L, z_{1}, z_{2}, \ldots, z_{n-1}\right\|_{n}}{\rho}\right)\right. \\
& \geq \varepsilon\} \in I .
\end{aligned}
$$

Since $\lim _{k \rightarrow \infty}$ inf $r_{k}>0$, it follows that

$$
\begin{aligned}
& \left\{\lim _{n} \frac{1}{\lambda_{n}} \sum_{k \in I_{n}}\left\{M\left(\frac{\left\|\Delta_{p}^{q} x_{\sigma^{k}(m)}-L, z_{1}, z_{2}, \ldots, z_{n-1}\right\|_{n}}{\rho}\right)\right\}^{r_{k}}\right. \\
& \geq \varepsilon\} \in I .
\end{aligned}
$$

And consequently, $x_{k} \rightarrow L \in\left[V_{\sigma}, \lambda, \Delta_{p}^{q}, M, r\right]^{I}$.

Let $\lim _{k \rightarrow \infty} r_{k}=r>0$. Suppose that $x_{k} \rightarrow L_{1} \in$ $\left[V_{\sigma}, \lambda, \Delta_{p}^{q}, M, r\right]^{I}, x_{k} \rightarrow L_{2} \in\left[V_{\sigma}, \lambda, \Delta_{p}^{q}, M, r\right]^{I}$, and $\left(\left\|L_{1}-L_{2}, z_{1}, z_{2}, \ldots, z_{n-1}\right\|_{n}\right)^{r_{k}}=a>0$.

Now, from (22) and the definition of Orlicz, we have

$$
\begin{aligned}
& \frac{1}{\lambda_{n}} \lim _{k \in I_{n}} M\left(\frac{\left\|L_{1}-L_{2}, z_{1}, z_{2}, \ldots, z_{n-1}\right\|_{n}}{\rho}\right)^{r_{k}} \\
& \leq \frac{C}{\lambda_{n}} \sum_{k \in I_{n}} M\left(\frac{\left\|\Delta_{p}^{q} x_{\sigma^{k}(m)}-L_{1}, z_{1}, z_{2}, \ldots, z_{n-1}\right\|_{n}}{\rho}\right)^{r_{k}} \\
& \quad+\frac{C}{\lambda_{n}} M\left(\frac{\left\|\Delta_{p}^{q} x_{\sigma^{k}(m)}-L_{2}, z_{1}, z_{2}, \ldots, z_{n-1}\right\|_{n}}{\rho}\right)^{r_{k}},
\end{aligned}
$$


since

$$
\begin{aligned}
& \{n \in N: \\
& \frac{1}{\lambda_{n}} \sum_{k \in I_{n}} M\left(\frac{\left\|\Delta_{p}^{q} x_{\sigma^{k}(m)}-L_{1}, z_{1}, z_{2}, \ldots, z_{n-1}\right\|_{n}}{\rho}\right)^{r_{k}} \\
& \geq \varepsilon\} \in I, \\
& \{n \in N: \\
& \frac{1}{\lambda_{n}} \sum_{k \in I_{n}} M\left(\frac{\left\|\Delta_{p}^{q} x_{\sigma^{k}(m)}-L_{2}, z_{1}, z_{2}, \ldots, z_{n-1}\right\|_{n}}{\rho}\right)^{r_{k}} \\
& \geq \varepsilon\} \in I \text {. }
\end{aligned}
$$

Hence,

$$
\left\{n \in N: \frac{1}{\lambda_{n}} \sum_{k \in I_{n}} M\left(\frac{\left\|\mathrm{E}_{1}-L_{2}, z_{1}, z_{2}, \ldots, z_{n-1}\right\|_{n}}{\rho}\right)^{r_{k}} \geq \varepsilon\right\} \in I .
$$

Further, $M\left(\left\|L_{1}-L_{2}, z_{1}, z_{2}, \ldots, z_{n-1}\right\|_{n} / \rho\right)^{r_{k}} \rightarrow M(a / \rho)^{r}$ as $k \rightarrow \infty$, and therefore

$$
\lim _{n \rightarrow \infty} \frac{1}{\lambda_{n}} \sum_{k \in I_{n}} M\left(\frac{\left\|L_{1}-L_{2}, z_{1}, z_{2}, \ldots, z_{n-1}\right\|_{n}}{\rho}\right)^{r_{k}}=M\left(\frac{a}{\rho}\right)^{r} .
$$

From (27) and (28), it follows that $M(a / \rho)=0$ and by the definition of an Orlicz function, we have $a=0$.

Hence, $L_{1}=L_{2}$ and this completes the proof.

Theorem 3. (i) Let $0<\inf _{k} r_{k} \leq r_{k} \leq 1$. Then,

$$
\left[V_{\sigma}, \lambda, \Delta_{p}^{q}, M, r\right]^{I} \subset\left[V_{\sigma}, \lambda, \Delta_{p}^{q}, M\right]^{I} .
$$

(ii) Let $0<r_{k} \leq \sup _{k} r_{k}<\infty$. Then,

$$
\left[V_{\sigma}, \lambda, \Delta_{p}^{q}, M\right]^{I} \subset\left[V_{\sigma}, \lambda, \Delta_{p}^{q}, M, r\right]^{I} .
$$

Theorem 4. Let $X\left(V_{\sigma}, \lambda, \Delta_{p}^{q-1}\right)$ stand for $\left(\left[V_{\sigma}, \lambda, \Delta_{p}^{q-1}, M, r\right]_{0}^{I}\right.$, $\left.\|\cdot, \cdot, \cdots\|_{n}\right),\left(\left[V_{\sigma}, \lambda, \Delta_{p}^{q-1}, M, r\right],\|\cdot, \cdot, \cdots\|_{n}\right)$, or $\left(\left[V_{\sigma}, \lambda \Delta_{p}^{q-1}, M\right.\right.$, $\left.r]_{\infty}^{I},\|\cdot, \cdot, \cdots\|_{n}\right)$ and $m \geq 1$. Then the inclusion $X\left(V_{\sigma}, \lambda\right.$, $\left.\Delta_{p}^{q-1}\right) \subset X\left(V_{\sigma}, \lambda, \Delta_{p}^{q}\right)$ is strict. In general, $X\left(V_{\sigma}, \lambda, \Delta_{p}^{i}\right) \subset$ $X\left(V_{\sigma}, \lambda, \Delta_{p}^{q}\right)$ for all $i=1,2,3, \ldots, p-1$ and the inclusion is strict.

Proof. Let us take $\left(\left[V_{\sigma}, \lambda, \Delta_{p}^{q-1}, M, r\right]_{0}^{I},\|\cdot, \cdot, \cdots\|_{n}\right)$.
Let $x=\left(x_{k}\right) \in\left(\left[V_{\sigma}, \lambda, \Delta_{p}^{q-1}, M, r\right]_{0}^{I},\|\cdot, \cdot, \cdots\|_{n}\right)$. Then for given $\varepsilon>0$, we have

$$
\begin{aligned}
& \{n \in N: \\
& \quad \frac{1}{\lambda_{n}} \sum_{k \in I_{n}}\left\{M\left(\frac{\left\|\Delta_{p}^{q-1} x_{\sigma^{k}(m)}, z_{1}, z_{2}, \ldots, z_{n-1}\right\|_{n}}{\rho}\right)\right\}^{r_{k}} \\
& \quad \geq \varepsilon\} \in I,
\end{aligned}
$$

for some $\rho>0$.

Since $M$ is nondecreasing and convex, it follows that

$\frac{1}{\lambda_{n}} \sum_{k \in I_{n}}\left\{M\left(\frac{\left\|\Delta_{p}^{q} x_{\sigma^{k}(m)}, z_{1}, z_{2}, \ldots, z_{n-1}\right\|_{n}}{\rho}\right)\right\}^{r_{k}}$

$=\frac{1}{\lambda_{n}}$

$\times \sum_{k \in I_{n}}\left\{M\left(\frac{\left\|\Delta_{p}^{q-1} x_{\sigma^{k+1}(m)}-\Delta_{p}^{q-1} x_{\sigma^{k}(m)}, z_{1}, z_{2}, \ldots, z_{n-1}\right\|_{n}}{\rho}\right)\right\}^{r_{k}}$

$\leq D \frac{1}{\lambda_{n}} \sum_{k \in I_{n}}\left(\left[\frac{1}{2} M\left(\frac{\left\|\Delta_{p}^{q-1} x_{\sigma^{k+1}(m)}, z_{1}, z_{2}, \ldots, z_{n-1}\right\|_{n}}{\rho}\right)\right]^{r_{k}}\right.$

$\left.+\left[\frac{1}{2} M\left(\frac{\left\|\Delta_{p}^{q-1} x_{\sigma^{k}(m)}, z_{1}, z_{2}, \ldots, z_{n-1}\right\|_{n}}{\rho}\right)\right]^{r_{k}}\right)$

$\begin{aligned} \leq D \frac{1}{\lambda_{n}} \sum_{k \in I_{n}}( & {\left[M\left(\frac{\left\|\Delta_{p}^{q-1} x_{\sigma^{k+1}(m)}, z_{1}, z_{2}, \ldots, z_{n-1}\right\|_{n}}{\rho}\right)\right]^{r_{k}} } \\ + & {\left.\left[M\left(\frac{\left\|\Delta_{p}^{q-1} x_{\sigma^{k}(m)}, z_{1}, z_{2}, \ldots, z_{n-1}\right\|_{n}}{\rho}\right)\right]^{r_{k}}\right) . }\end{aligned}$

Hence we have

$$
\begin{aligned}
& \{n \in N: \\
& \left.\frac{1}{\lambda_{n}} \sum_{k \in I_{n}}\left\{M\left(\frac{\left\|\Delta_{p}^{q} x_{\sigma^{k}(m)}, z_{1}, z_{2}, \ldots, z_{n-1}\right\|_{n}}{\rho}\right)\right\}^{r_{k}} \geq \varepsilon\right\}
\end{aligned}
$$




$$
\begin{aligned}
& \subseteq\{n \in N: \\
& D \frac{1}{\lambda_{n}} \sum_{k \in I_{n}}\left\{M\left(\frac{\left\|\Delta_{p}^{q-1} x_{\sigma^{k+1}(m)}, z_{1}, z_{2}, \ldots, z_{n-1}\right\|_{n}}{\rho}\right)\right\}^{r_{k}} \\
& \left.\geq \frac{\varepsilon}{2}\right\} \\
& \cup\{n \in N: \\
& D \frac{1}{\lambda_{n}} \sum_{k \in I_{n}}\left\{M\left(\frac{\left\|\Delta_{p}^{q-1} x_{\sigma^{k}(m)}, z_{1}, z_{2}, \ldots, z_{n-1}\right\|_{n}}{\rho}\right)\right\}^{r_{k}} \\
& \left.\geq \frac{\varepsilon}{2}\right\} \text {. }
\end{aligned}
$$

Since the set on the right hand side belongs to $I$, so does the left hand side. The inclusion is strict as the sequence $x=\left(k^{r}\right)$, for example, belongs to $\left(\left[V_{\sigma}, \lambda, \Delta_{p}^{q}, M\right]_{0}^{I},\|\cdot, \cdot, \cdots\|_{n}\right)$ but does not belong to $\left(\left[V_{\sigma}, \lambda, \Delta_{p}^{q-1}, M\right]_{0}^{I},\|\cdot, \cdot, \cdots\|_{n}\right)$ for $M(x)=x$ and $r_{k}=1$ for all $k$.

Theorem 5. $\left(\left[V_{\sigma}, \lambda, \Delta_{p}^{q}, M, r\right]_{0}^{I},\|\cdot, \cdot, \cdots\|_{n}\right)$ and $\left(\left[V_{\sigma}, \lambda, \Delta_{p}^{q}\right.\right.$, $\left.M, r]^{I},\|\cdot, \cdot, \cdots\|_{n}\right)$ are complete linear topological spaces, with paranorm $g$, where $g$ is defined by

$$
\begin{aligned}
& g(x) \\
& =\sum_{m=1}^{p q}\left\|x_{\sigma^{k}(m)}, z_{1}, z_{2}, \ldots, z_{n-1}\right\|_{n} \\
& +\inf \left\{\rho^{r_{k} / H}:\right. \\
& \left.\quad \sup _{m, n}\left(\frac{1}{\lambda_{n}} \sum_{k \in I_{n}}\left(M\left(\frac{\left\|\Delta_{p}^{q} x_{\sigma^{k}(m)}, z_{1}, z_{2}, \ldots, z_{n-1}\right\|_{n}}{\rho}\right)\right)\right)^{H}\right\},
\end{aligned}
$$

where $H=\max \left(1,\left(\sup _{k} r_{k}\right)\right)$.

\section{Conflict of Interests}

The authors declare that there is no conflict of interests regarding the publication of this paper.

\section{Acknowledgments}

The first author acknowledges that this research was part of the research project and partially supported by the Universiti Putra Malaysia under Grant no. ERGS 1-2013/5527179. The work of second author was carried out under the Postdoctoral Fellow under National Board of Higher Mathematics, DAE, Project no. NBHM/PDF.50/2011/64.

\section{References}

[1] G. G. Lorentz, "A contribution to the theory of divergent sequences," Acta Mathematica, vol. 80, pp. 167-190, 1948.

[2] I. J. Maddox, "Spaces of strongly summable sequences," The Quarterly Journal of Mathematics, vol. 18, pp. 345-355, 1967.

[3] P. Schaefer, "Infinite matrices and invariant means," Proceedings of the American Mathematical Society, vol. 36, pp. 104-110, 1972.

[4] M. Mursaleen, "Matrix transformations between some new sequence spaces," Houston Journal of Mathematics, vol. 9, no. 4, pp. 505-509, 1983.

[5] P. Kostyrko, T. Salat, and W. Wilczynski, "On I-convergence," Real Analysis Exchange, vol. 26, no. 2, pp. 669-685, 2001.

[6] J. Lindenstrauss and L. Tzafriri, "On Orlicz sequence spaces," Israel Journal of Mathematics, vol. 10, pp. 379-390, 1971.

[7] H. Kizmaz, "On certain sequence spaces," Canadian Mathematical Bulletin, vol. 24, no. 2, pp. 169-176, 1981.

[8] S. Gähler, "Lineare 2-normierte Räume," Mathematische Nachrichten, vol. 28, pp. 1-43, 1964.

[9] A. Misiak, " $n$-inner product spaces," Mathematische $\mathrm{Na}$ chrichten, vol. 140, pp. 299-319, 1989.

[10] M. Gurdal, "On ideal convergent sequences in 2-normed spaces," Thai Journal of Mathematics, vol. 4, no. 1, pp. 85-91, 2006.

[11] M. Gurdal and A. Sahiner, "Ideal convergence in $n$-normed spaces and some new sequence spaces via $n$-norm," Journal of Fundamental Sciences, vol. 4, no. 1, pp. 233-244, 2008. 


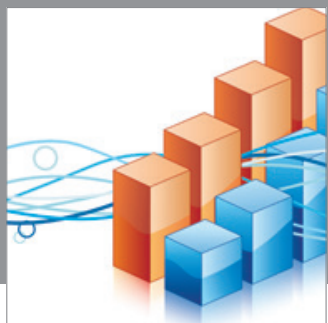

Advances in

Operations Research

mansans

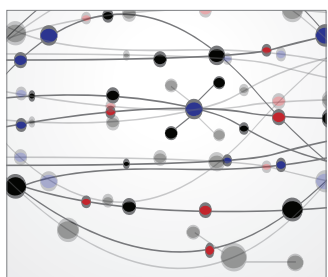

The Scientific World Journal
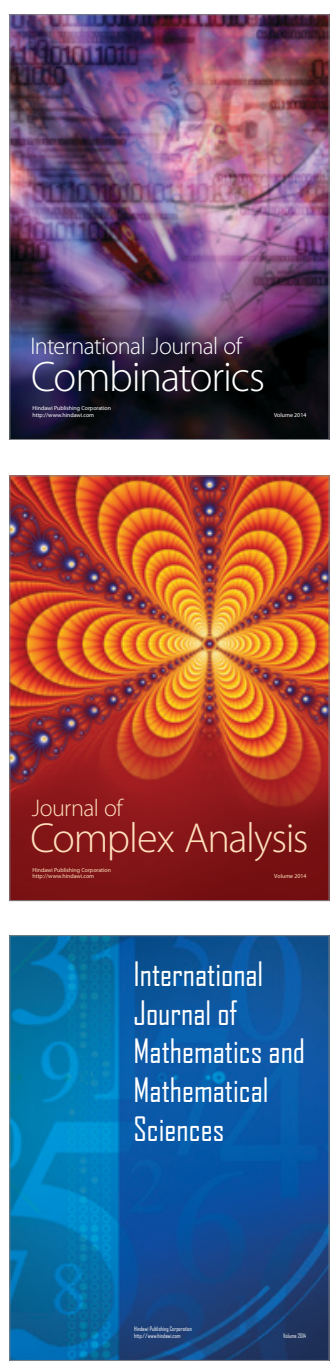
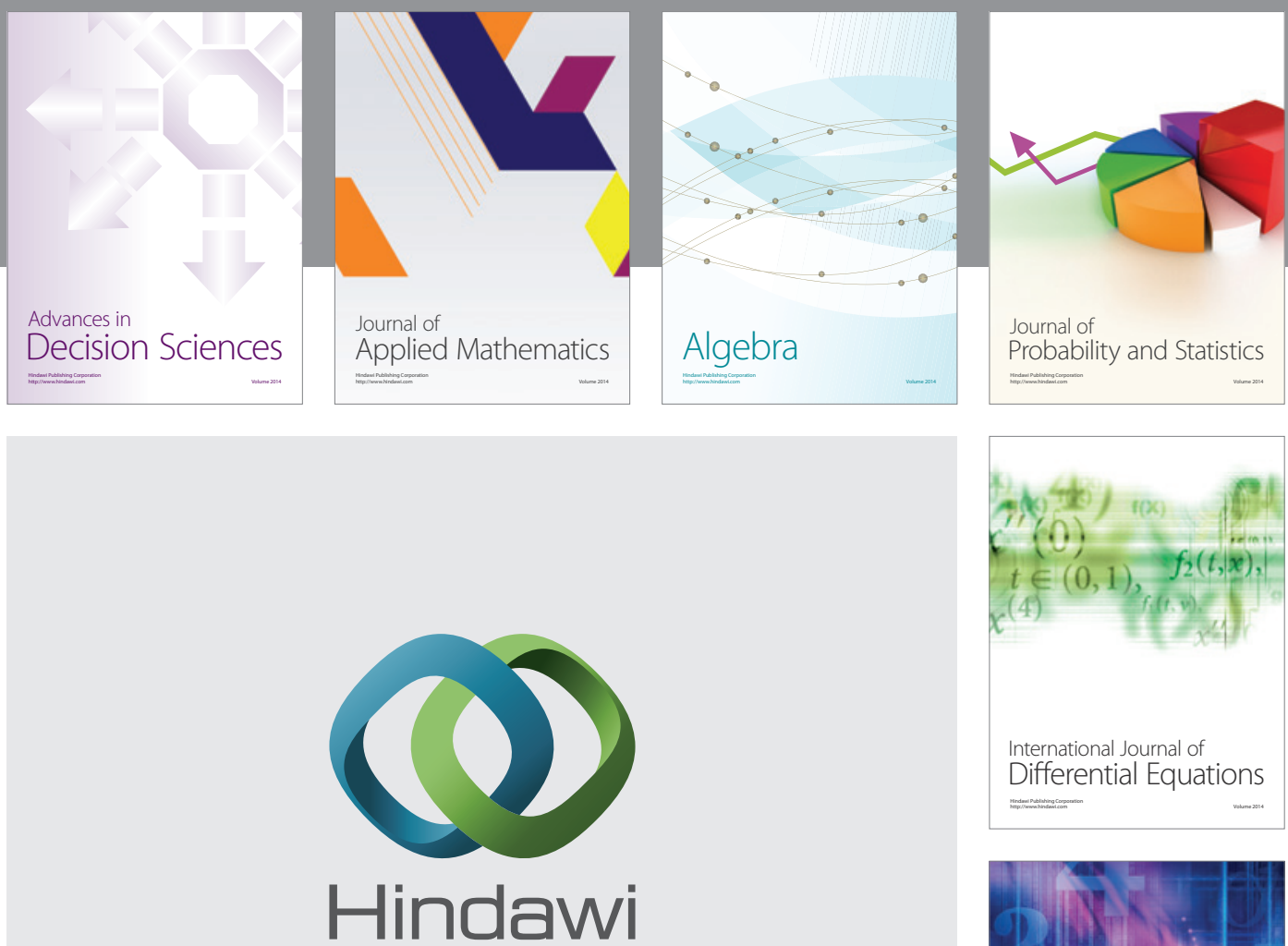

Submit your manuscripts at http://www.hindawi.com
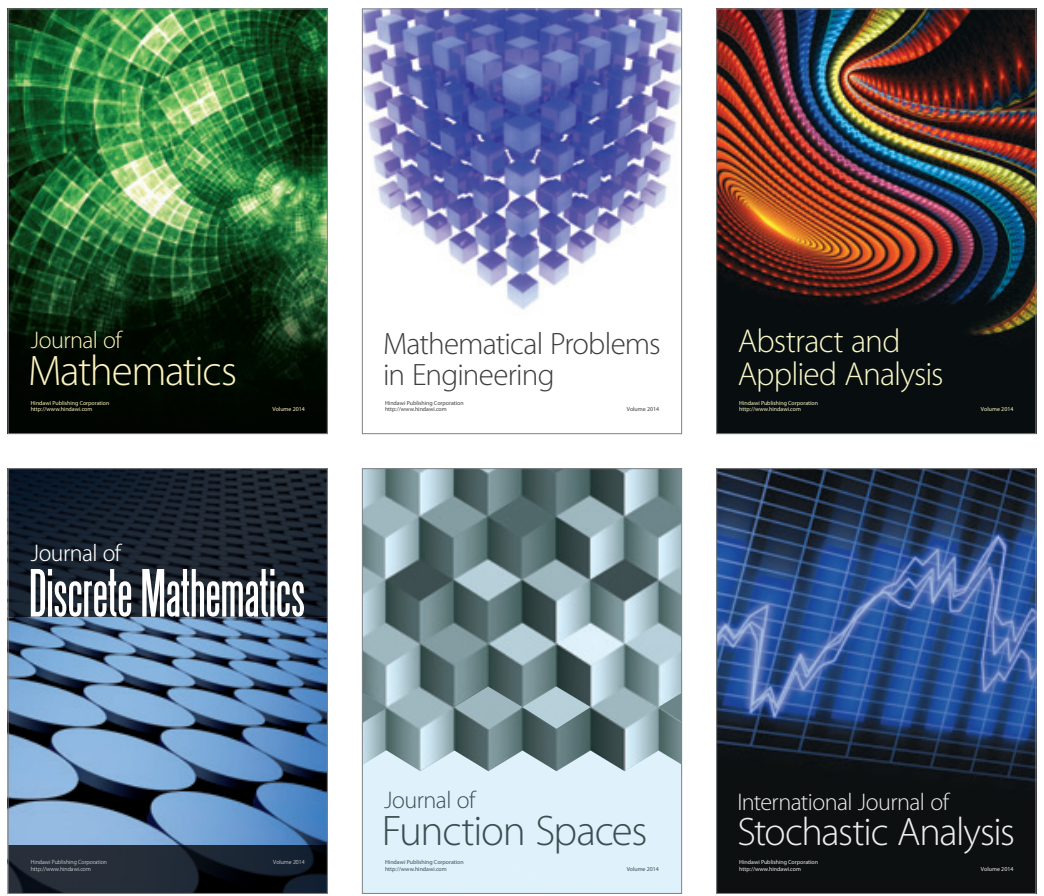

Journal of

Function Spaces

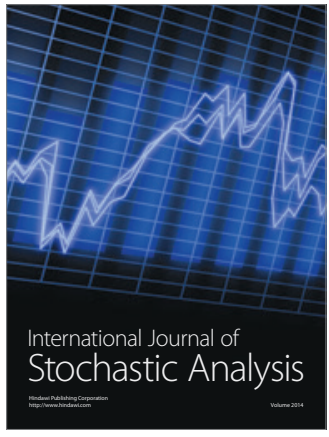

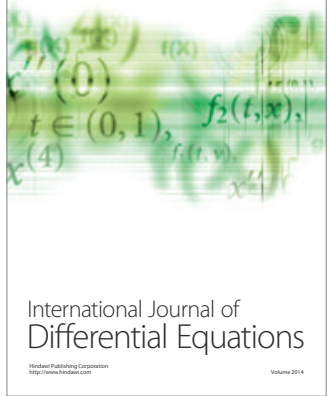
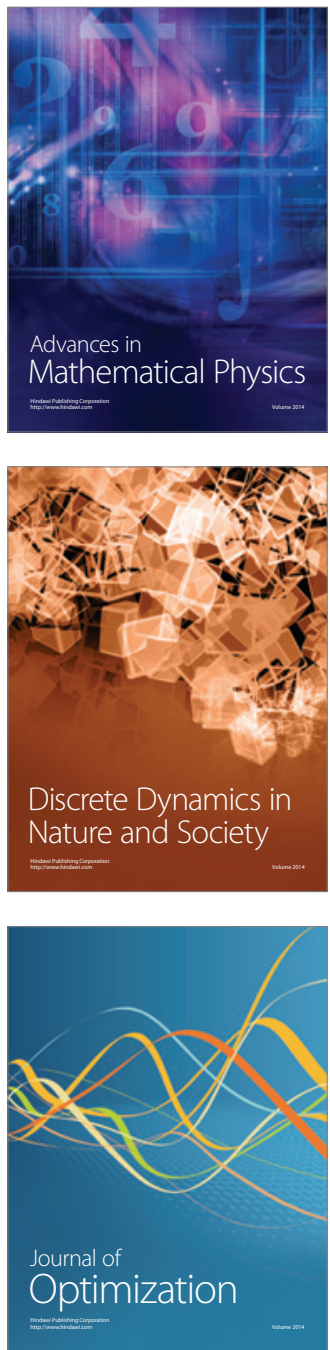